\title{
Babies born to mothers positive for SARS-CoV-2 - Are they in danger?
}

\author{
Joon Kee Lee, MD, $\mathrm{PhD}^{1,2}$
}

${ }^{1}$ Department of Pediatrics, Chungbuk National University Hospital, Cheongju, Korea; ${ }^{2}$ Department of Infection Control and Prevention, Chungbuk National University Hospital, Cheongju, Korea

\section{Key Message}

Concerns have arisen in the coronavirus disease 2019 (COVID-19) pandemic upon pregnancy and postnatal care starting from reproductive decision-making. To the utmost knowledge, reproductive decisions should not be based primarily on health-related COVID-19 concerns, as the possibility of vertical transmission is negligible and the perinatal outcome is generally not poor compared to pregnancies without COVID-19, as long as infection control measures are well kept.

Amid the coronavirus disease 2019 (COVID-19) pandemic, life goes on and new babies are born. Relatively mild disease and the high proportion of asymptomatic infections in children and adolescents with COVID-19 offer some degree of relief, even though these facts themselves increase infection control difficulties in communities and health care centers. ${ }^{1)}$ Aside from the favorable course of COVID-19 in the pediatric population, we, as pediatricians, are involved with some pregnancy and postnatal care issues. Therefore, the challenging situation of the pandemic should not be disregarded.

Numerous questions regarding pregnancy and COVID-19 have been raised, ultimately starting from reproductive decisionmaking ("Shall $\mathrm{I} / \mathrm{we}$ plan to conceive during this pandemic?"). Many questions remain unanswered and a large portion cannot be answered easily, which means that shared decisions should be made using interim knowledge and available guidelines. Of major concern is the possibility of vertical transmission and the perinatal outcome in mothers who test positive for severe acute respiratory syndrome coronavirus 2 (SARS-CoV-2). Considerable studies have been conducted on these specific issues, and even though more studies need to be completed, studies to date assist with the shared decision-making of families and attending physicians. In this issue of Clinical and Experimental Pediatrics, Sharma et al..$^{2}$ have explored these problems by an ambispective observational study that examined 44 neonates born to 41 SARS-CoV-2 positive mothers from North India. The results of the study showed no definite direct adverse effect on the fetus or newborn (perinatal outcome), and a negligible possibility of vertical transmission.

The main results of the study are in line with similar studies, some of which include systematic reviews and meta-analyses. ${ }^{3,4)}$ In addition, to date, SARS-CoV-2 infections in neonates are generally reported as case reports or series, in which mortality directly and solely associated with SARS-CoV-2 infection was scarce. Rather, enthusiasm for vigorously investigating the biological samples including cord blood, placental membrane swab, vaginal fluid, amniotic fluid, peritoneal fluid (in case of caesarean section), and breast milk should be recognized. Owing to the design of the study of Sharma et al.,2) the authors were able to collect the specimens from 28 mothers. Still, a few studies have discovered positive SARS-CoV-2 in some of the biological samples enumerated above. For example, most placentas studied so far had no evidence of infection. However, evidence of the virus in placentas has been identified in other studies. ${ }^{5)}$ Even so, we should keep in mind that proving nonexistence takes much more effort than proving existence. Therefore, the added evidence by the authors cannot be devalued.

Interesting results of the study of Sharma et al..$^{2)}$ included comparably high percentages of preterm deliveries (14.6\%) and caesarean sections (56.1\%) (Fig. 1). Preterm delivery is often considered when the mother has pneumonia and when the delivery would help the course of COVID-19. Regardless, the majority of the mothers in this study did not have moderate to severe COVID-19-related conditions. Therefore, it would be helpful to know if the authors presented the usual rate of preterm births in the institution. In addition, many relevant studies are from developed countries; the conditions may be quite different in developing countries in terms of preterm delivery. Referentially, the overall preterm delivery rates in the United States from two large cohort studies were $7.2 \%$ to $12.9 \% .6,7)$

A high rate of caesarean section has been repeatedly reported globally since the initial phases of the pandemic. Due to the nature of the virus, when moderate to severe pneumonia develops it is natural to think labor with maximum effort is

Corresponding author: Joon Kee Lee, MD, PhD. Department of Pediatrics, Chungbuk National University Hospital, 776 1-Sunhwan-ro, Seowon-gu, Cheongju 28644, Korea 


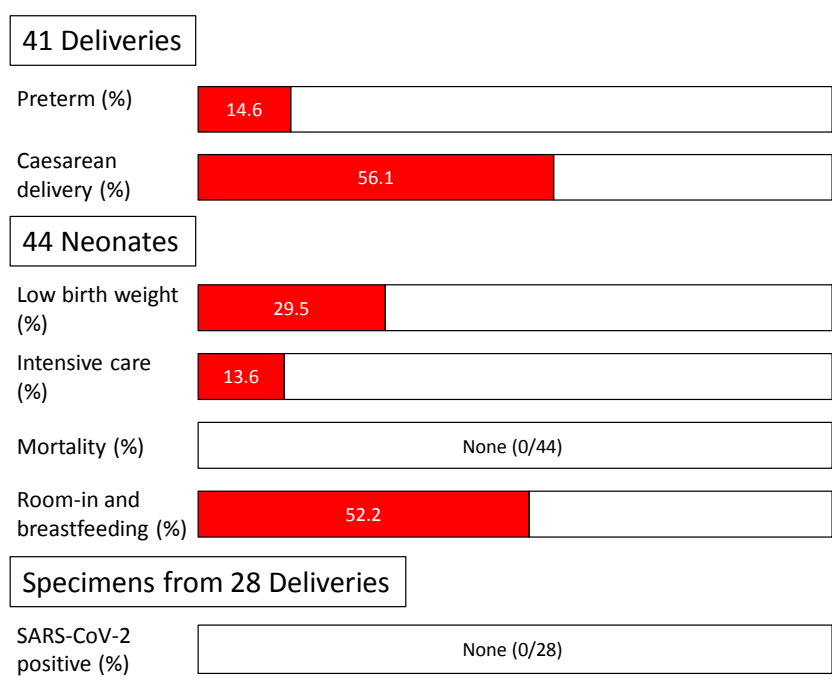

Fig. 1. Summary of the outcomes of 41 deliveries, 44 neonates, and severe acute respiratory syndrome coronavirus 2 (SARS-CoV-2) results of specimens collected from 28 deliveries.

impossible. Therefore, the increased proportion may be perceived hypothetically as natural. Nevertheless, consequentially, the majority of the caesarean deliveries were not because of the above issue, as many studies reported an elevated rate of caesarean delivery overall. Vaginal birth usually takes longer than caesarean delivery. This might have been the driving force for increased caesarean delivery rates in the initial phases of the pandemic in terms of infection control and decreasing vertical transmission of the virus. This is partly endorsed by experience with human immunodeficiency virus. ${ }^{8)}$ Fortunately, as studies show better outcome in the newborn with vaginal birth compared to caesarean delivery, the rates are decreasing. ${ }^{9)}$

Finally, the study also emphasizes the safety and importance of rooming-in and breastfeeding, even though 30\% of the mothers agreed to rooming-in and breastfeeding in the specific facility. Theoretically, the mother and newborn may be deprived of memorable and rare life moments when decisions are made based on misinformation. Nevertheless, regardless of the degree, the fear of COVID-19 infection is realistic and practical for the family. Considering this, the age-old issue of the conflict between evidence-based medicine and family-centered care could be reiterated. ${ }^{10)}$ The entire study reminds us of the obligations of pediatricians to be knowledgeable, up-to-date, and to make their best effort in shared decision-making with families. Furthermore, if possible, pediatricians themselves should add any gathered evidence to the literature regarding COVID-19, however small.
Conflict of interest

No potential conflicts of interest relevant to this article are reported.

See the article "Perinatal outcome and possible vertical transmission of coronavirus disease 2019: experience from North India" via https://doi.org/10.3345/cep.2020.01704.

\section{References}

1. Han MS, Choi EH, Chang SH, Jin BL, Lee EJ, Kim BN, et al. Clinical characteristics and viral RNA detection in children with coronavirus disease 2019 in the Republic of Korea. JAMA Pediatr 2021;175:73-80.

2. Sharma R, Seth S, Sharma R, Yadav S, Mishra P, Mukhopadhyay S. Perinatal outcome and possible vertical transmission of coronavirus disease 2019: experience from North India. Clin Exp Pediatr 2021 Feb. DOI: 10.3345/cep.2020.01704.

3. Allotey J, Stallings E, Bonet M, Yap M, Chatterjee S, Kew T, et al. Clinical manifestations, risk factors, and maternal and perinatal outcomes of coronavirus disease 2019 in pregnancy: living systematic review and meta-analysis. BMJ 2020;370:m3320.

4. Kotlyar AM, Grechukhina O, Chen A, Popkhadze S, Grimshaw A, Tal O, et al. Vertical transmission of coronavirus disease 2019: a systematic review and meta-analysis. Am J Obstet Gynecol 2021;224:35-53.e3.

5. Penfield CA, Brubaker SG, Limaye MA, Lighter J, Ratner AJ, Thomas $\mathrm{KM}$, et al. Detection of severe acute respiratory syndrome coronavirus 2 in placental and fetal membrane samples. Am J Obstet Gynecol MFM 2020;2:100133.

6. Mullins E, Hudak ML, Banerjee J, Getzlaff T, Townson J, Barnette K, et al. Pregnancy and neonatal outcomes of COVID-19: co-reporting of common outcomes from PAN-COVID and AAP SONPM registries. Ultrasound Obstet Gynecol 2021;57:573-81.

7. Woodworth KR, Olsen EOM, Neelam V, Lewis EL, Galang RR, Oduyebo $\mathrm{T}$, et al. Birth and infant outcomes following laboratory-confirmed SARSCoV-2 infection in pregnancy - SET-NET, 16 jurisdictions, March 29October 14, 2020. MMWR Morb Mortal Wkly Rep 2020;69:1635-40.

8. Read JS, Newell ML. Efficacy and safety of cesarean delivery for prevention of mother-to-child transmission of HIV-1. Cochrane Database Syst Rev 2005;(4):CD005479.

9. Martinez-Perez O, Vouga M, Cruz Melguizo S, Forcen Acebal L, Panchaud A, Munoz-Chapuli M, et al. Association between mode of delivery among pregnant women with COVID-19 and maternal and neonatal outcomes in Spain. JAMA 2020;324:296-9.

10. Siminoff LA. Incorporating patient and family preferences into evidencebased medicine. BMC Med Inform Decis Mak 2013;13 Suppl 3:S6.

How to cite this article: Lee JK. Babies born to mothers positive for SARS-CoV-2 - Are they in danger? Clin Exp Pediatr 2021;64:280-1. https://doi.org/10.3345/cep.2021.00276 